\title{
DBSA doped polypyrrole blended with Poly(4-styrenesulfonic acid) by mechanical mixing
}

\author{
P. Jayamurugan ${ }^{1,2}$, V. Ponnuswamy ${ }^{1 *}$, S. Ashokan, R. N. JAYAPraKaSh ${ }^{2}$, N. AShOK ${ }^{2}$, \\ K. GUNA ${ }^{2}$, R. MARIAPPAN ${ }^{1,2}$ \\ ${ }^{1}$ Department of Physics, Sri Ramakrishna Mission Vidyalaya College of Arts and Science, Coimbatore-20, Tamilnadu, India \\ ${ }^{2}$ Department of Physics, Adhiyamaan College of Engineering, Hosur-635109, Tamilnadu, India
}

DBSA doped polypyrrole was prepared by In-situ chemical oxidative polymerization method. The reaction temperature was 0 to $20{ }^{\circ} \mathrm{C}$. Different weight percentages of PSS (40 wt. $\%, 60 \mathrm{wt} \%$ and $80 \mathrm{wt} . \%$ ) were mechanically blended with a pestle in an agate mortar for 25 minutes by solid state mixing. The investigation of the blend focused on the optical, structural and morphological properties. SEM micrographs indicated that PSS was homogeneously distributed within DBSA doped PPy. FT-IR study confirmed the doped and blended dopants in the composite structure. UV-study revealed the $\pi \rightarrow \pi^{*}$ transition in benzenoid rings of DBSA and presence of PSS. The semi-crystalline nature of the composites improved with increasing the weight percentage of PSS.

Keywords: solid-state mixing; composites; morphological; structural and optical properties

(C) Wroclaw University of Technology.

\section{Introduction}

Polypyrrole (PPy) is one of the most frequently studied polymers due to its environmental stability, relative ease of synthesis, and good electrical conductivity. PPy is the most commonly used in commercial applications, such as batteries, super capacitors, sensors and corrosion protection [1].

The applicability of ICPs, such as polypyrrole, is restricted by their poor process ability and the resulting mechanical properties. PPy, however, can be processed without altering its structure by blending with some conventional polymers. Mixing of polymers has become commercially and technologically more important than synthesis of homo-polymers and copolymers in the last decade. Blending allows creating new materials with specific properties for the desired application at low cost. These blends are designed to combine the desired properties of both components, i.e. electrical conductivity of PPy along with process ability and physical properties of the matrix polymer.

*E-mail: muruganjaya85@gmail.com
The blends of polymers were prepared chemically by in situ polymerization [2] solution blending [3] colloidal dispersion [4] and melt processing [5] and also by electrochemical methods. Youqing Shen et al. [6] reported solution blending of polypyrrole by mechanical treatment using organic acid. It is also well known that DBSA doped polypyrrole, blended with various concentrations of PSS by solid state mixing, have not been reported until now. The present study focuses on investigation of DBSA doped PPy, blended with PSS by solid state mixing. The effect of PSS on DBSA doped polypyrrole has been studied.

\section{Experimental}

\subsection{Synthesis of DBSA doped polypyrrole}

Ammonium persulphate (APS) obtained from Loba Chemicals, methanol and acetone (Merck) were used for the synthesis. Pyrrole and dodecylbenzene sulfonic acid (DBSA) (Sigma-Alridch, India) were purified by using distillation plant. $0.1 \mathrm{~mol}$ DBSA and $0.3 \mathrm{~mol}$ of Pyrrole were dissolved in $500 \mathrm{ml}$ of deionized water. After 
30 minutes, $0.06 \mathrm{~mol}$ of ammonium persulfate (APS) was dissolved in $100 \mathrm{ml}$ deionized water and added dropwise into the solution mixture. The solution was stirred for 24 hours in an ice bath maintained at a constant temperature of 0 to $5{ }^{\circ} \mathrm{C}$. The color of the solution became dark brown. $300 \mathrm{ml}$ of methanol was added to solution to stop the reaction. The precipitate was collected and washed with deionized water, methanol and acetone and the samples were dried at $30{ }^{\circ} \mathrm{C}$ for 12 hours in a vacuum oven.

\subsection{Solid-state blending of polypyr- role/PSS composites}

Poly(4-styrenesulfonic acid) (PSS MW 75,000) was purchased from (Sigma-Alridch, India). DBSA doped polypyrrole was prepared by chemical oxidative polymerization method. The different weight percentages of PSS (40 wt.\%, 60 wt. $\%$ and 80 wt.\%) were mechanically blended with a pestle in an agate mortar for 25 minutes by solid state blending at room temperature. The optical, structural and morphological studies were carried out for the prepared composite powder.

\section{Characterization techniques}

FT-IR analysis was done in the range of 4000 to $400 \mathrm{~cm}^{-1}$ using Thermo Nicolet V-200 FT-IR spectrometer by $\mathrm{KBr}$ pellet technique. An X-ray diffractometer (XPERTPRO) with $\mathrm{Cu} \mathrm{K} \alpha$ radiation $\lambda=15.4 \mathrm{~nm}$ was used to identify the crystalline nature of the polypyrrole and its composites samples. AJEOL JSM-6390 scanning electron microscope was used to study the surface morphology of DBSA-PPy blended with PSS composite powder. The UV-Vis spectra of DBSA doped polypyrrole with different weight percentages of PSS were recorded employing Jasco V-530 dual beam spectrometer.

\section{Results and discussion}

\subsection{FT-IR spectra of solid-state blended polypyrrole/PSS composites}

FT-IR spectra of solid-state blended PPy/PSS composites are shown in Fig. 1(a-c). The peaks at
1545,1397 and $1450 \mathrm{~cm}^{-1}$ are due to $\mathrm{C}=\mathrm{C}$ stretching vibration of pyrrole. The weak band that appears at $1682 \mathrm{~cm}^{-1}$ is attributed to $\mathrm{C}=\mathrm{O}$ groups in the composite structure. The peak at $874 \mathrm{~cm}^{-1}$ is due to doping state of polypyrrole. The band at $447 \mathrm{~cm}^{-1}$ is due to $\mathrm{C}-\mathrm{S}$ stretching vibrations. This implies that the sulfonic acid groups were introduced into the polymer back bone.

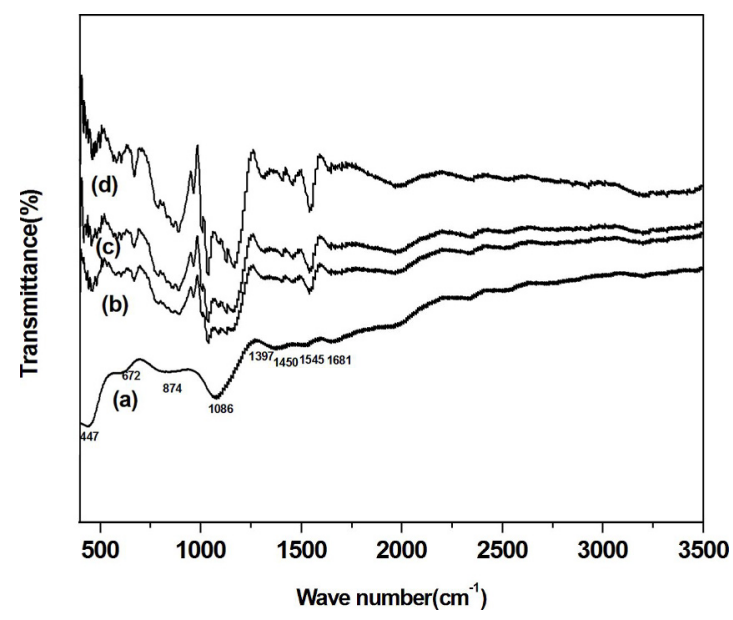

Fig. 1. FT-IR spectra of solid-state blended PPy/PSS composites: (a) DBSA doped PPY; (b) 40 wt.\%; (c) 60 wt. \%; (d) 80 wt.\%.

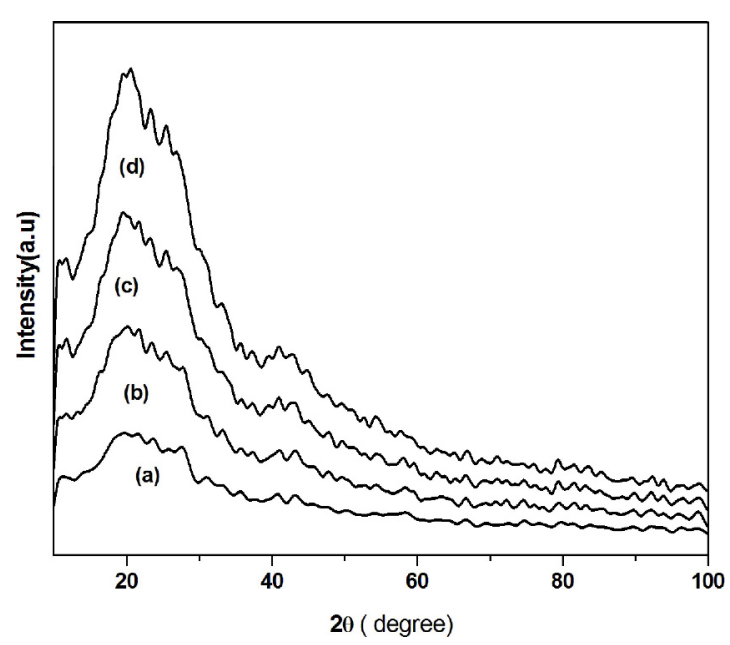

Fig. 2. X-ray diffraction of solid-state blended PPy/PSS composites: (a) DBSA doped PPY; (b) $40 \mathrm{wt} . \%$; (c) 60 wt. \%; (d) 80 wt.\%.

The absorption peaks at 1037 and $1170 \mathrm{~cm}^{-1}$ are associated with pure PSS. The higher 

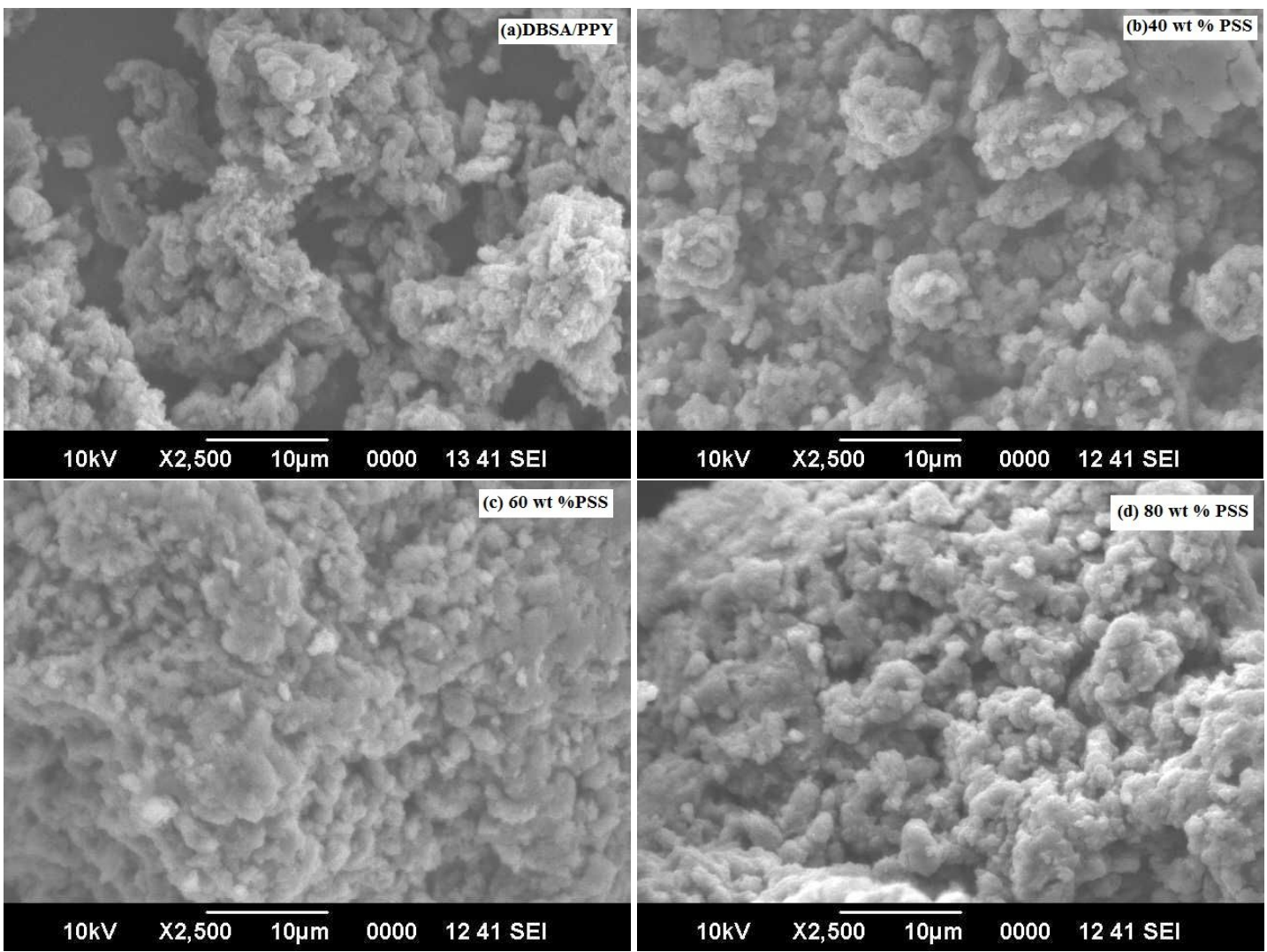

Fig. 3. SEM images of DBSA doped polypyrrole, blended with different weight percentages of PSS.

percentage of PSS was confirmed by the increased intensity of the composites. We observed that the PSS was present in the composite structure.

\subsection{X-ray diffraction of solid-state blended polypyrrole/PSS composites}

Fig. 2 shows the XRD patterns of DBSA doped PPy, PPy/PSS composites. The XRD results reveal broad amorphous diffraction peaks in the range from $2 \theta=11^{\circ}$ to $24^{\circ}$ for DBSA doped PPy and its composites. The PSS without polypyrrole shows a broad peak and the higher concentration of PSS reveals the semi-crystalline nature of the composite powders. The high concentrations of $\mathrm{SO}_{3}$-groups make changes in the PPy matrix. PSS has good interaction with DBSA doped PPy. It is observed that higher concentration of PSS shows semi-crystalline nature of the composite powders.

\subsection{SEM images of DBSA doped polypyr- role blended with PSS}

Fig. 3 shows the SEM images of DBSA doped PPy blended with different weight percentages of PSS.

Fig. $3 \mathrm{a}$ and $3 \mathrm{~b}$ shows the particle phase separation. No phase separation is observed in Fig. $3 \mathrm{c}$ and $3 \mathrm{~d}$, which shows good compatibility of the components. It is seen from the micrographs that the mixture of PSS with DBSA doped PPy is homogeneous. This homogeneous mixture of PSS may improve the conductivity of polypyrrole composites. The smoothness of blend surfaces increases with increasing the sulfonation degree. It is observed that the samples containing higher weight percentage of PSS show homogeneous mixture of phases and good compatibility with DBSA doped polypyrrole. 
4.4. UV-Vis absorption for different weight percentages of PSS and DBSA doped polypyrrole composites

Fig. 4 shows absorption spectra of DBSA doped polypyrrole with different weight ratios of PSS. The absorption peak at $230 \mathrm{~nm}$ reveals $\pi \rightarrow \pi *$ transition in benzenoid rings of DBSA and presence of PSS.

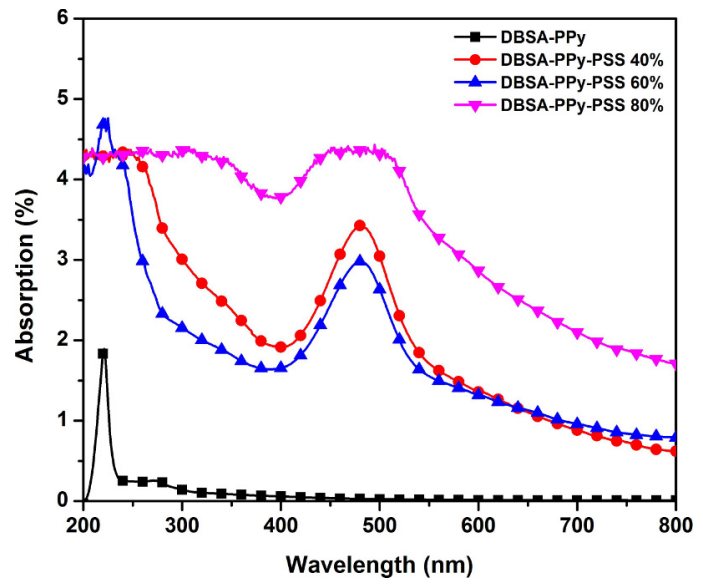

Fig. 4. UV-Vis absorption spectra of DBSA doped polypyrrole, blended with PSS.

The band at $497 \mathrm{~nm}$ shows the repeating units of PSS chains [7]. The band at $230 \mathrm{~nm}$ disappears at $80 \%$ of polystryenesulfonic acid. This band indicates the absence of DBSA and the higher blending level of PSS with polypyrrole.

\section{Conclusion}

DBSA doped polypyrrole was prepared by Insitu chemical oxidative polymerization method.
The different weight percentages of PSS (40 wt.\%, $60 \mathrm{wt} . \%$ and $80 \mathrm{wt} . \%$ ) were mechanically blended with a pestle in an agate mortar for 25 minutes by solid state mixing. FT-IR spectra confirm the structural changes of prepared composites. XRD patterns show semi-crystalline nature of the composite at higher weight percentages of PSS. SEM images reveal homogeneous mixing of phases and good compatibility with PSS blended polypyrrole. UV-study indicates the presence DBSA at low concentration but the absence of DBSA at higher concentration of PSS.

\section{Acknowledgements}

The authors are thankful to sophisticated test and instrumentation center, Cochin (Kerala), and Birla Institute of Technology, Mesra, Ranchi for providing instrumental facilities.

\section{References}

[1] Deivanayaki S., Ponnuswamy V., Jayamurugan P., ASHOKAn S., Elixir Polymer., 49B (2012), 1018210185.

[2] Bhat N V., Joshi N V., J. Appl Polym Sci., 50(1993), 1423.

[3] Pron A., Osterholm J E., Smith P., Heeger AJ., LASKA J., ZAGORSKA M., Synth. Met., 57(1993), 3520.

[4] BANERJEe P., MANDAL BM., Macromolecules, 28(1995), 3940.

[5] Shacklette W W., Han CC., Luly M H., Synth. Met., 57(1993), 3532.

[6] Youqing Shen., Meixiang., J. Appl. Polym. Sci., 68(1998), 1277-1284.

[7] Zotti G., Zecchin S., Schiavon G., Louwet F., Groenendaal L., Crispin X., Osikowicz W., SALANECK M., FAHLMAN, Macromolecules, 36 (2003), 3337-3344. 